BULL. AUSTRAL. MATH. SOC.

$46 A 32,46 A 20,54 A 05$

VOL. $21(1980), 281-301$.

(46M05)

\title{
CONVERGENCE TENSOR PRODUCTS \\ AND A STRICT TOPOLOGY
}

\author{
Bernd MüLler
}

\begin{abstract}
We are interested in the strict topology $\tau$ on $L_{s}(E, E)$, the set $L(E, F)$ of all continuous linear mappings from $E$ into a Banach space $F$ endowed with the topology of pointwise convergence. The $T_{3}$-completion $E \hat{\otimes}_{c} L_{c} F$ of the convergence tensor product $E \otimes_{c} L_{c} F$ is the set of all $\tau$-continuous linear functionals on $L(E, F)$ and $\tau$ is the topology of uniform convergence on the compact subsets of $E \hat{\otimes}_{c} L_{c} F$. In the case that $E$ is a nuclear Fréchet space, a nuclear $(D F)$-space or a Banach space with the bounded approximation property the topology $\tau$ agrees with the topology of $L_{C o}(E, F)$.
\end{abstract}

Let $E$ be a locally convex topological vector space and $F$ a Banach space. We are interested in the finest locally convex vector space topology $\tau$ on the set $L(E, F)$ of all continuous linear mappings from $E$ to $F$ such that every filter $\Phi$ on $L(E, F)$ converges to 0 with respect to $\tau$, if it has the following properties:

(i) $\Phi$ converges pointwise to 0 ;

(ii) $\Phi$ contains a pointwise bounded subset of $L(E, F)$. This topology $\tau$ is called the strict topology on $L_{s}(E, F)$. If $E$ is barrelled the Banach-Steinhaus theorem says that $\tau$ is finer than the

Received 25 October 1979. 
topology of $L_{C o}(E, F)$, the set $L(E, F)$ endowed with the topology of precompact convergence. The continuous convergence structure is the finest convergence structure $\gamma$ on $L(E, F)$ such that every filter with the. properties ( $i$ ) and (ii) $\gamma$-converges to 0 . Therefore $\tau$ is the finest locally convex vector space topology coarser than the continuous convergence structure. Let us denote by $L_{c}(E, F)$ and $\left(L_{c}(E, F)\right)_{\tau}$ the set $L(E, E)$ carrying the continuous convergence structure and the finest locally convex vector space topology coarser than the continuous convergence structure. It is known that this topology is the topology of uniform convergence on the compact subsets of $L_{c}\left(L_{c}(E, F)\right)$, the dual of $L_{C}(E, F)$ also endowed with the continuous convergence structure. For this reason we show in Section 2: the $T_{3}$-completion $E \hat{\otimes}_{c} L_{c} F$ of the convergence tensor product $E \otimes_{c} L_{c} E$ is isomorphic to $L_{c}\left(L_{c}(E, F)\right)$. In some cases we will prove $\left(L_{c}(E, F)\right)_{\tau}=L_{c o}(E, F)$, for example, if $E$ is a nuclear Fréchet space, a nuclear $(D F)$-space or a Banach space with the bounded approximation property. But we will also prove

$$
\left(L_{c}\left(\mathbb{R}^{D}, \tau_{1}\right)\right)_{\tau} \neq L_{c o}\left(\mathbb{R}^{D}, \tau_{1}\right)
$$

where $R^{D}$ is an uncountable product of $R$ and $l_{1}$ is the Banach space of all real sequences which are absolutely summable. This example shows that $L_{C O}(E, F)$ does not always carry the finest locally convex vector space topology such that every filter with the properties (i) and (ii) converges to 0 .

Since, in this paper, the concept of convergence spaces will be used, let us say something about this concept. Every convergence space will be a convergence space in the sense of Fischer (see [1]). For a subset $S$ of a convergence space $X$, the adherence $a(S)$ is defined to be the set

$$
\{y: y \in X \text {, there exists a filter } \Phi \text { converging to } y \text { with } S \in \Phi\} \text {. }
$$

$S$ is called dense in $X$ if $a(S)=X$, compact if every ultra-filter $\Phi$ with $S \in \Phi$ converges in $S$, and relatively compact if $a(S)$ is compact. A mapping $f$ from a convergence space $X$ into a convergence space $Y$ is continuous if for all $x \in X$ and all filters $\Phi$ converging to $x$ in $X$ 
the filter $f(\Phi)$ generated by $\{f(L): L \in \Phi\}$ converges to $f(x)$ in $Y$. Let us denote by $\mathcal{C}_{C}(X, Y)$ the set $\mathcal{C}(X, Y)$ of all continuous mappings from $X$ into $Y$ endowed with the continuous convergence structure. This convergence structure is the coarsest on $\mathcal{C}(X, Y)$ for which the mapping $\omega: \mathcal{C}(X, Y) \times X \rightarrow Y$ defined by $\omega(f, x)=f(x)$ for all $x \in X$ and $f \in \mathcal{C}(X, Y)$, is continuous. This means that a filter $F$ converges to $f$ in $\mathcal{C}_{c}(X, Y)$ if and only if for all $x \in X$ and all filters $\Phi$ converging to $x$ in $X$ the filter $\omega(F, \Phi)$ converges to $f(x)$ in $Y$. Instead of $\omega(F, \Phi)$ we will also write $F(\Phi)$.

All vector spaces considered in this paper will be R-vector spaces. A vector space space endowed with a convergence structure is called a convergence vector space if the algebraic operations are continuous. For a convergence vector space $E$ we will denote by $E_{\tau}$ the set $E$ endowed with the finest locally convex vector space topology coarser than the convergence structure of $E$. A subspace $F$ of a convergence vector space $E$ will be always a vector subspace carrying the convergence structure induced by $E$. A $T_{3}$-convergence vector space $E$ will be a separated convergence vector space such that for every filter $\Phi$ converging to 0 in $E$ the filter $a(\Phi)$ generated by $\{a(V): V \in \Phi\}$ converges also to 0 . Let $E, F$ and $G$ be convergence vector spaces. A bijective linear mapping $T: E \rightarrow F$ is called an isomorphism if $T$ and $T^{-1}$ are continuous. Let us denote by $L_{c}(E, F)$ and $B_{c}(E, F ; G)$ the subspaces of $C_{C}(E, F)$ and $C_{C}(E \times F, G)$ respectively consisting of the set $L(E, F)$ of all continuous linear mappings from $E$ into $E$ and of the set $B(E, F ; G)$ of all continuous bilinear mappings from $E \times F$ into $G$ respectively. We abbreviate $B_{c}(E, F ; R), B(E, F ; R), L_{c}(E, R)$ and $L(E, R)$ to $B_{c}(E, F), B(E, F), L_{c}(E)$ or $L_{c} E$ and $L E$ respectively. For every convergence vector space $E$ there exists a natural mapping $j: E \rightarrow L_{c} L_{c} E$ defined by $[j(x)](\zeta)=\zeta(x)$ for all $x \in E$ and $\zeta \in L E$, which is always continuous. $E$ is called $L_{c}$-embedded if $j$ is an isomorphism from $E$ onto $j(E)$, and c-reflexive if $j$ is an isomorphism from $E$ onto $L_{c} L_{c} E$. For every $L_{c}$-embedded convergence vector space $E$ 
we will identify $E$ and $j(E)$. Let us mention that every subspace of an $L_{c}$-embedded convergence vector space is also $L_{c}$-embedded.

Let $X$ and $Y$ be vector spaces and $\langle X, Y\rangle$ a duality (see [15]). For a subset $A$ of $X$ we denote by $\bar{A}^{-}$the closure of $A$ with respect to the topology $\sigma(X, Y)$, by $A^{0}$ the polar of $A$ which is defined as $A^{0}=\{y: y \in Y,|y(a)| \leq 1$ for all $a \in A\}$ and by $\Gamma A$ the convex, circled hull of $A$. Let us mention that $(L(E, F), E \otimes L F\rangle$ is a duality, if $E$ and $F$ are separated locally convex topological vector spaces. This duality is defined by $T(x \otimes \zeta)=\zeta(T(x))$ for all $x \in E, \zeta \in L F$ and $T \in L(E, F)$.

\section{Convergence tensor products}

Since $\left(L_{C}(E, F)\right)_{\tau}$ carries the topology of uniform convergence on the compact subsets of $L_{c}\left(L_{c}(E, F)\right)$, we will develop a duality theory for $L_{C}(E, F)$. For this reason we are interested in convergence tensor products. For convergence vector spaces $E$ and $F$ let us denote by $E \otimes_{L} F$ the tensor product of $E$ and $F$ in the category of all convergence vector spaces. The existence of tensor products in this category was proved by several authors, for example, by Antoine [1] or Jarchow [10]. The convergence structure on $E \otimes_{L} F$ is the finest convergence vector space structure for which the canonical mapping $X: E \times F \rightarrow E \otimes F$ defined by $\chi(x, y)=x \otimes y$, is continuous. As usual we write $U \otimes V$ instead of $\chi(U, V)$ for subsets $U$ and $V$ of $E$ and $F$ respectively. Throughout this paper we assume that $E$ and $F$ are convergence vector spaces with point-separating duals. Therefore the mapping $j: E \otimes F \rightarrow L_{C} L_{C}\left(E \otimes_{L} F\right)$ is injective. Let us denote by $E \otimes_{C} F$ the tensor product of $E$ and $F$ endowed with the convergence structure induced by the mapping $j$.

We shall need the following lemma.

LEMMA I.1. Let $E$ be a convergence vector space, $F$ an $L_{c}$-embedded convergence vector space. Denote by $E_{c}$ the vector space $E$ endowed with the convergence structure induced by $L_{c} L_{c} E$. Then 
$L_{c}(E, F)=L_{c}\left(E_{c}, E\right)$.

Proof. $L_{c}(E, F)$ is a subspace of $L_{c}\left(E, L_{c} L_{c} E\right)$ which is isomorphic to $B_{c}\left(E, L_{c} F\right)$ (see [3]). But $B_{c}\left(E, L_{c} F\right)$ is a subspace of $C_{c}\left(E \times L_{c} F\right)$ which is c-reflexive (see [5]). Therefore $L_{c}(E, F)$ is $L_{c}$-embedded. Denote by $E_{L}$ the vector space $E$ considered as a subspace of $L_{c}\left(L_{c}(E, F), F\right)$. Then $E_{L}$ is $L_{c}$-embedded, $L(E, F)=L\left(E_{L}, F\right)$ and the evaluation mapping $\omega: L_{c}(E, F) \times E_{L} \rightarrow F$ defined by $\omega(T, x)=T(x)$ for all $x \in E$ and $T \in L(E, F)$, is continuous. Since $L_{c}\left(E_{L}, F\right)$ carries the coarsest convergence structure for which $\omega$ is continuous, the identity mapping $I: L_{c}(E, F) \rightarrow L_{c}\left(E_{L}, F\right)$ is continuous. This implies $L_{c}(E, F)=L_{c}\left(E_{C}, F\right)=L_{c}\left(E_{L}, F\right)$ since the convergence structure of $E_{C}$ is finer than that of $E_{L}$ and coarser than that of $E$.

THEOREM I.2. The convergence space $E \otimes_{c} F$ has the following properties:

(i) $E \otimes_{c} F$ carries the finest $L_{c}$-embedded convergence structure for which the canonical mapping $X: E \times F \rightarrow E \otimes F$ is continuous;

(ii) $B_{c}(E, F ; G)$ and $L_{c}\left(E \otimes_{c} F, G\right)$ are isomorphic for every $L_{c}$-embedded convergence vector space $G$;

(iii) if $E$ and $F$ are locally convex topological vector spaces we have $E \otimes_{c} F=E \otimes_{\pi} F$, where $E \otimes_{\pi} F$ is the projective tensor product of $E$ and $F$.

Proof. $E \otimes_{c} F$ carries the finest $L_{c}$-embedded convergence structure which is coarser than the convergence structure of $E \otimes_{L} F$. This implies (i).

(ii). Let us define $\alpha: L_{c}\left(E \otimes_{c} F, G\right) \rightarrow B_{c}(E, F ; G)$ by $\alpha(T)=T \circ X \cdot \alpha$ is continuous since $X$ is. In [10] it is shown that $\alpha$ is a bijective mapping. The filters which are finite sums of filters of 
the form $\chi(x+U, v)$ and $\chi(u, y+V)$ where $x \in E, y \in F$ and $u$ and $v$ are filters converging to 0 in $E$ and $F$ respectively, generate the ideal of all filters converging to 0 in $E \Theta_{L} F$ (see [10]). Therefore $\alpha^{-1}$ is a continuous mapping from $B_{c}(E, F ; G)$ onto $L_{c}\left(E \otimes_{L} F, G\right)$. Lemma $I . I$ now implies that $B_{c}(E, F ; G)$ and $L_{c}\left(E \otimes_{c} F, G\right)=L_{c}\left(E \otimes_{L} F, G\right)$ are isomorphic.

(iii). Let $U$ and $V$ be the o-neighborhood filters in $E$ and $E$ respectively. $\chi(U, V)$ converges to 0 in $E \otimes_{c} F$ and, since $E \otimes_{c} F$ is $L_{c}$-embedded, the filter generated by $\{\Gamma(U \otimes V): U \in U, V \in U\}$ also converges to 0 . This implies $E \otimes_{\mathrm{C}} F=E \otimes_{\pi} F$.

Since $L_{c}\left(E, L_{c}(F, G)\right)$ and $B_{c}(E, F ; G)$ are always isomorphic (see [1] and [3]) we conclude:

COROLLARY 1. $L_{c}\left(E \otimes_{c} E, G\right)$ and $L_{c}\left(E, L_{c}(F, G)\right)$ are isomorphic for all convergence vector spaces $E$ and $F$ and for all $L_{c}$-embedded convergence vector spaces $G$. If $E$ is a c-reflexive convergence vector. space, $L_{c}\left(E \otimes_{c} L_{c} F\right)$ and $L_{c}(E, F)$ are isomorphic. For convenience, let us write $L_{c}\left(E \otimes_{c} L_{c} F\right)=L_{c}(E, F)$ if $F$ is c-reflexive.

COROLLARY 2. If $F$ is c-reflexive and if $E \otimes L F$ is dense in $L_{c}\left(L_{c}(E, F)\right)$, then the convergence vector space $L_{c}(E, F)$ is c-reflexive.

Proof.

$$
\begin{aligned}
L(E, F) & =L\left(E \otimes_{c} L_{c} F\right)=L\left(L_{c} L_{c}\left(E \otimes_{c} L_{c} F\right)\right) \\
& =L\left(L_{c}\left(L_{c}(E, F)\right)\right) .
\end{aligned}
$$

REMARK. Another way to introduce $E \otimes_{c} F$ is suggested by Binz: consider $E \otimes F$ as a subspace of $L_{c}\left(B_{c}(E, F)\right)$. Then by Theorem I.2 this convergence vector space agrees with $E \otimes_{c} F$. 


\section{Locally convex topological vector spaces}

From now on, $E$ and $F$ are always assumed to be separated locally convex topological vector spaces. We denote by $L_{s}(E, F)$ and $L_{c o}(E, F)$ the set $L(E, F)$ endowed with the topology of pointwise and of precompact convergence respectively which is always coarser than the continuous convergence structure. Instead of $L_{C O}(E, R)$ we write $L_{C O}(E)$. In order to apply Corollary 1 of Theorem I.2, we also assume that $E$ and $F$ are complete, since $F$ is c-reflexive if and only if it is complete (see [5]).

In [13] a complete $T_{3}$-convergence vector space $\hat{H}$ is called the $T_{3}$-completion of $H$, if $\hat{H}$ has the following properties:

(i) there is an isomorphism $i$ from $H$ onto a subspace of $\hat{H}$;

(ii) for every complete $T_{3}$-convergence vector space $M$ and for every $T \in L(H, M)$ there exists a $\hat{T} \in L(\hat{H}, M)$ with $T=\hat{T} \circ i$.

In this section we will show that $L_{c}\left(L_{c}(E, F)\right)$ is the $T_{3}$-completion of $E \otimes_{c} L_{c} F$ if $F$ is a Banach space. Further on we will show $\left(L_{C}(E, F)\right)_{\tau}=L_{c o}(E, F)$ if and only if, for every compact subset $C$ of $L_{c}\left(L_{c}(E, F)\right)$ there exist compact subsets $K_{1}$ and $K_{2}$ of $E$ and $L_{c} F$ respectively such that $c \subseteq a\left(\Gamma\left(K_{1} \otimes K_{2}\right)\right)$.

Let $F$ be a Banach space, $U$ the O-neighborhood filter in $E$ and $\Phi$ a filter in $L(E, F)$. Since $\Phi(U)$ converges to 0 in $F$ if and only if $\Phi$ contains an equicontinuous set we get (see also [2]):

LEMMA II.1. If $F$ is a Banach space, a filter $\Phi$ converges to 0 in $L_{c}(E, F)$ if and only if

(i) $\Phi$ converges to 0 in $L_{s}(E, F)$,

(ii) $\Phi$ contains an equicontinuous subset.

LEMMA II.2. $E \otimes L F$ is dense in $L_{c}\left(L_{c}(E, F)\right)$ for every Banach 
space $F$.

Proof. Let $H$ be the family of all equicontinuous convex, circled and pointwise closed subsets of $L(E, F)$. By Lemma II.l the continuous convergence structure and the topology of pointwise convergence agree on every $H \in H$. Therefore every $\zeta \in L\left(L_{c}(E, F)\right)$ is continuous on $H$ with respect to the topology of pointwise convergence. This implies that for all $0<\varepsilon \in \mathbf{R}$ one can find an $f \in L\left(L_{s}(E, F)\right)=E \otimes L F$ with $(\zeta-f)(H) \subseteq[-\varepsilon, \varepsilon]$ (see [15], Chapter IV, Theorem 6.2). Define $D_{H, \varepsilon}:=\{f: f \in E \otimes L F,(\zeta-f)(H) \subseteq[-\varepsilon, \varepsilon]\}$. We will show that the filter $F$ generated by $\left\{D_{H, \varepsilon}: H \in H, O<\varepsilon \in \mathbf{R}\right\}$ converges to $\zeta$ in $L_{c}\left(L_{c}(E, F)\right)$. Choose an $\varepsilon>0$ and let $\Phi$ be a filter converging to $T$ in $L_{c}(E, F)$. Since $\zeta$ is continuous, one can find an equicontinuous subset $G \in \Phi$ with $\zeta(G)-\zeta(T) \subseteq[-E, E]$. Choose an $H \in H$ with $G \subseteq H$. Then we have

$$
D_{H, \varepsilon}(g)-\zeta(T) \subseteq\left[D_{H, \varepsilon}(g)-\zeta(g)\right]+[\zeta(g)-\zeta(T)] \subseteq[-2 \varepsilon, 2 \varepsilon]
$$

for all $g \in G$. Therefore $E \otimes L F$ is dense in $L_{c}\left(L_{c}(E, F)\right)$.

LEMMA II.3. Let $F$ be a Banach space. For every fizter $F$ converging to 0 in $L_{c}\left(L_{c}(E, F)\right)$ there exists a fizter $G$ in $E \otimes L F$ with the following properties:

(i) the fizter $\hat{G}$ generated by $\{a(G): G \in G\}$ is coarser than $F$, where $a(G)$ denotes the adherence of $G$ in $L_{c}\left(L_{c}(E, F)\right)$;

(ii) $\hat{G}$ converges to 0 in $L_{c}\left(L_{c}(E, F)\right)$.

Proof. For every $0<\varepsilon \in \mathbb{R}$ and for every filter $\Phi$ converging to $T$ in $L_{c}(E, F)$ there exists an $H_{\Phi} \in \Phi$ with $\varepsilon\left(H_{\Phi}\right)^{0} \in F$, where the polar is taken with respect to the duality $\left\langle L(E, F), L L_{c}(E, F)\right\rangle$. By Lemma II.1, we can assume that $H_{\Phi}$ belongs to the family $H$ of all equicontinuous, convex, circled and pointwise closed subsets of $L(E, F)$. The filter $N$ generated by the filter subbase 


$$
\left\{\left(\frac{1}{\varepsilon} H_{\Phi}\right)^{0}: 0<\varepsilon \in \mathbf{R}, \Phi \text { converges in } L_{c}(E, F)\right\}
$$

is coarser than $F$ and converges to 0 in $L_{c}\left(L_{c}(E, F)\right)$. We now show:

$$
\bigcap_{i=1}^{n}\left(\frac{1}{\varepsilon_{i}} H_{\Phi_{i}}\right)^{0} \subseteq a\left(2 \bigcap_{i=1}^{n}\left(\frac{1}{\varepsilon_{i}} H_{\Phi_{i}}\right)^{0} \cap(E \otimes L F)\right)
$$

for all $n \in \mathbb{N}$, where the adherence is taken in $L_{c}\left(L_{c}(E, F)\right)$. The set

$$
K=\left(\bigcap_{i=1}^{n}\left(\frac{1}{\varepsilon_{i}} H_{\Phi_{i}}\right)^{0}\right)^{0}
$$

belongs to $H$. As proved in Lemma II.2, for every

$$
\zeta \in K^{0}=\bigcap_{i=1}^{n}\left(\frac{1}{\varepsilon_{i}} H_{\Phi_{i}}\right)^{0}
$$

and for every $H \in H$ one can find an $x_{H} \in E \otimes L F$ with $\zeta-x_{H} \in H^{0}$ such that the net $\left(x_{H}\right)_{H \in H}$ converges to $\zeta$ in $L_{c}\left(L_{c}(E, F)\right)$. For all $H \in H$ with $K \subseteq H$ we have

$$
x_{H}=x_{H}-\zeta+\zeta \in H^{0}+K^{0} \subseteq 2 K^{0} .
$$

Therefore the filter $G$ generated in $E \otimes L F$ by

$$
\left\{2\left(\frac{1}{\varepsilon} H_{\Phi}\right)^{0} \cap(E \otimes L F): 0<\varepsilon \in \mathbb{R}, \Phi \text { converges in } L_{c}(E, F)\right\}
$$

has the desired property.

THEOREM II.4. Let $F$ be a Banach space. Then we have:

(i) $L_{c}\left(L_{c}(E, F)\right)$ is the $T_{3}$-completion $E \hat{\otimes}_{c} L_{c} F$ of $E \otimes_{c} L_{c} F$;

(ii) $\left(L_{c}(E, F)\right)_{\tau}$ carries the topology of uniform convergence on the compact subsets of $E \hat{\otimes}_{c} L_{c} F$.

Proof. By definition, $E \otimes_{c} L_{c} F$ is a subspace of

$$
L_{c} L_{c}\left(E \otimes_{c} L_{c} F\right)=L_{c}\left(L_{c}(E, F)\right) \text {. }
$$


Let $M$ be a complete $T_{3}$-convergence vector space and $T \in L\left(E \otimes_{c} L_{c} F, M\right)$. For every $\zeta \in L\left(L_{c}(E, F)\right)$ choose a filter $F$ on $E \otimes L F$ converging to $\zeta$ in $L_{c}\left(L_{c}(E, F)\right)$. Define $\hat{T}(\zeta)$ to be the limit of the filter $T(F)$ in $M$. Lemma II.3 implies the continuity of $T$, since for every filter $G$ converging to 0 in $E \otimes_{c} L_{c} F$ the filter $a(T(G))$ is coarses than $\hat{T}(a(G))$.

To prove the second part use the results of [6].

LEMMA II.5. Let $U$ be a subset of $E$ and $V$ a closed, convex, circled subset of $F$. Define

$$
T_{U, V}=\{T: T \in L(E, F), T(U) \subseteq V\}
$$

Then we have

$$
\left.T_{U, V}^{0}=\overline{\Gamma\left(U \otimes V^{0}\right)^{\sigma}} \text { and } \overline{\left(\Gamma\left(U \otimes V^{0}\right)^{\sigma}\right.}\right)^{0}=T_{U, V}
$$

where the polars of $T U, V$ and $V$ are taken with respect to the dualities $\left\langle L L_{C}(E, F), L(E, F)\right)$ and $(F, L F\rangle$ respectively, and $\sigma$ denotes the weak topology $\sigma\left(L\left(L_{C}(E, F)\right), L(E, F)\right)$.

PROPOSITION II.6. $\left(L_{c}(E, F)\right)_{\tau}=L_{c u}(E, F)$ if and only if, for every compact subset $C$ of $L_{c}\left(L_{c}(E, F)\right)$, there exist compact subsets $K_{1}$ and $K_{2}$ of $E$ and $L_{c} E$ respectively such that

$$
c \subseteq a\left(\Gamma\left(K_{1} \otimes K_{2}\right)\right)
$$

Proof. Assume $\left(L_{C}(E, E)\right)_{\tau}=L_{C o}(E, F)$ and let $C$ be a compact subset of $L_{c}\left(L_{c}(E, F)\right)$. The polar $C^{0}$ of $C$, taken with respect to the duality $\left\langle L\left(L_{c}(E, F)\right), L(E, F)\right\rangle$ is a 0-neighborhood in $\left(L_{c}(E, F)\right)_{\tau}$ (to prove this use [6]). Therefore there exist a compact subset $K \subseteq E$ and a closed, circled, convex 0 -neighborhood $V$ in $F$ such that

$$
T_{K, V}=\{T: T \in L(E, F), T(K) \subseteq V\} \subseteq C^{0}
$$

$V^{0}$ and $\left(T_{K, V}\right)^{0}$ are equicontinuous and therefore compact, topological 
subsets of $L_{c} E$ and $L_{c}\left(L_{c}(E, F)\right)$ respectively (see [6]). Now Lemma II.5 implies

$$
c \subseteq c^{00} \subseteq\left(T_{K, V}\right)^{0}=\overline{\Gamma\left(K \otimes v^{0}\right)^{\sigma}}=a\left(\Gamma\left(K \otimes v^{0}\right)\right)
$$

To prove the converse let $W$ be a closed, convex, circled O-neighborhood in $\left(\mathrm{L}_{\mathrm{c}}(E, F)\right)_{\tau}$. Since $W^{0}$ is compact in $\mathrm{L}_{\mathrm{c}}\left(\mathrm{L}_{\mathrm{c}}(E, F)\right)$, one can find compact subsets $K_{1}$ and $K_{2}$ of $E$ and $L_{c} F$ respectively such that

$$
W^{0} \subseteq a\left(\Gamma\left(K_{1} \otimes K_{2}\right)\right) \subseteq a\left(\Gamma\left(K_{1} \otimes K_{2}^{00}\right)\right)
$$

Thus

$$
\begin{aligned}
a\left(\Gamma\left(K_{1} \otimes K_{2}^{00}\right)\right\}^{0} & =\left(\overline{\left(\Gamma K_{1} \otimes K_{2}^{00}\right)^{\sigma}}\right)^{0} \\
& =\left\{T: T \in L(E, F), T\left(K_{1}\right) \subseteq K_{2}^{0}\right\} \subseteq W .
\end{aligned}
$$

Since $K_{2}^{0}$ is a 0 -neighborhood in $F$, Proposition II.6 is proved.

The following was partially proved in [4].

THEOREM II.7. Let $E$ and $F$ be Fréchet spaces. Then $L_{\mathrm{CO}}\left(E, L_{\mathrm{CO}}(F)\right)$ carries the finest topology which is coarser than the convergence structures of $L_{c}\left(E, L_{c} F\right)$ and $L_{c}\left(E, L_{c o}(F)\right)$.

Proof. Let us first show $L\left(E, L_{c} E\right)=L\left(E, L_{c o}(F)\right)$. For every $T \in L\left(E, L_{c o}(F)\right)$ define a bilinear mapping $v_{T}: E \times E \rightarrow \mathrm{R}$ by $v_{T}(x, y)=[T(x)](y)$. Since $E$ and $F$ are Fréchet spaces, $v_{T}$ is continuous. Therefore one can find 0 -neighborhoods $U$ and $V$ in $E$ and $F$ respectively such that

$$
v_{T}(U, V)=[T(U)](V) \subseteq[-1,1],
$$

which implies that $T(U)$ is an equicontinuous subset of $L F$. Therefore $T$ is also a continuous mapping from $E$ into $L_{c} F$.

We have 


$$
L_{c}\left(L_{c}\left(E, L_{c} F\right)\right)=L_{c}\left(L_{c}\left(E \otimes_{\pi} F\right)\right)=E \hat{\otimes}_{\pi} F
$$

where $E \hat{\otimes}_{\pi} F$ is the completion of $E \otimes_{\pi} F$. By the Banach-Dieudonné theorem, the finest topology $t$ on $L\left(E \otimes_{\pi} F\right)$ coarser than the continuous convergence structure is the topology of uniform convergence on the compact subsets of $E \hat{\otimes}_{\pi} F$. Grothendieck has shown that to every compact subset $C$ of $E \hat{\otimes}_{\pi} F$ there exist compact, circled, convex subsets $K_{1}$ and $K_{2}$ of $E$ and $F$ respectively with $C \subseteq \overline{\Gamma\left(K_{1} \otimes K_{2}\right)^{\sigma}}$. Lemma II.5 implies

$$
\left(\Gamma\left(K_{1} \otimes K_{2}\right)\right)^{0}=T_{K_{1}, K_{2}^{0}}
$$

which is a O-neighborhood in $L_{c o}\left(E, L_{c o}(E)\right)$. Since the convergence structure of $L_{C}\left(E, L_{C O}(F)\right)$ is coarser than that of $L_{C}\left(E, L_{C}(F)\right)$ and finer than the topology of $L_{c o}\left(E, L_{c o}(E)\right)$, Theorem II.7 is proved.

COROLLARY. Let $E$ be a Eréchet space and $F$ a nuclear (DF)-space. Then $L_{\mathrm{Co}}(E, F)$ carries the finest topology which is coarser than the convergence structure of $L_{c}(E, F)$.

Proof. Every nuclear $(D F)$-space is a $k$-space, therefore a subset of $L_{C o}(F)$ is relatively compact if and only if it is equicontinuous. This implies $F=L_{C O}\left(L_{C O}(F)\right)$. Now $L_{C O}(F)$ is a Fréchet space and we can apply Theorem II.7.

LEMMA II.8. We have $\left(E \otimes_{c} L_{c} F\right)_{\tau}=E \otimes_{\pi} L_{c o}(F)$ if and only if for every equicontinuous subset $H \subseteq L(E, F)$ which is relatively compact in $L_{s}(E, F)$, there exists a 0-neighborhood $U$ in $E$ such that $H(U)$ is relatively compact in $F$.

Proof. A subset $H \subseteq L(E, F)$ is relatively compact in $L_{\mathrm{c}}(E, F)$ if and only if $H$ is equicontinuous and relatively compact in $L_{s}(E, F)$ (see [6]). The topology of $\left(E \otimes_{C} L_{c} E\right)_{\tau}$ is the topology of uniform convergence on the equicontinuous subsets of $L\left(\left(E \otimes_{c} L_{c} F\right)_{\tau}\right)$ which are the relatively 
compact subsets of $L_{c}\left(E \otimes_{c} L_{c} F\right)=L_{c}(E, F)$ (see [6]). Therefore we have $\left(E \otimes_{\mathrm{C}} L_{c} F\right)_{\tau}=E \otimes_{\pi} L_{C O}(F)$ if and only if for every compact subset $H$ of $L_{c}(E, F)$ there exist a 0-neighborhood $U$ in $E$ and a compact subset $K \subseteq F$ such that $\Gamma\left(U \otimes K^{0}\right) \subseteq H^{0}$ which, by Lemma II.5, is equivalent to $H \subseteq\left\{T: T \in L(E, E), T(U) \subseteq K^{00}\right\}$.

\section{Banach spaces}

Throughout this section we assume that $E$ and $F$ are Banach spaces. For a subset $D \subseteq L(E, F)$ let us denote by $\vec{D}$ the closure of $D$ in $L_{s}(E, F)$. We will always consider $E \otimes L F$ as a subset of $L_{c}(E, F)$. For every subset $B \subseteq E$ we define

$$
T_{B}=\{T: T \in L(E, F),\|T(b)\| \leq 1 \text { for all } b \in B\} \text {. }
$$

With the same methoas used in the proof of Lemma 2.4 in [11] one can show:

LEMMA III.1. Let $E$ be a Banach space, $G$ a subspace of $E$ with finite dimension $k$ and let $T \in L(E, E)$ be a compact operator. Assume that there exists an $\varepsilon \in \mathbf{R}$ with $0<\varepsilon<1$ and $k \varepsilon(1-\varepsilon)^{-1}<1$ such that $\|T(x)-x\| \leq \varepsilon\|x\|$ for alz $x \in G$.

Then there exists a compact operator $S \in L(E, E)$ such that

$$
S(x)=x \text { for all } x \in G \text { and }\|S\| \leq 2\|T\| \text {. }
$$

By Lemma II.I, it is easy to prove:

PROPOSITION III.2. The set $K(E, E)$ of all compact operators from $E$ into $E$ is dense in $L_{c}(E, E)$ if and only if there exists a net $\left(T_{\alpha}\right)_{\alpha \in J}$ in $K(E, E)$ with the following properties:

(i) $\left(T_{\alpha}(x)\right)_{\alpha \in J}$ converges to $x$ for alz $x \in E$;

(ii) there exists an $n \in R, 0<n$ with $\left\|T_{\alpha}\right\|<n$ for all $\alpha \in J$.

Let us remember that, for Banach spaces $E$ and $F$, a filter $\Phi$ converges to 0 in $L_{c}(E, F)$ if and only if it converges to 0 in 
$L_{s}(E, F)$ and there is an $n \in N$ with $n T_{U} \in \Phi$, where $U$ is the closed unit ball in $E$. Therefore the continuous convergence structure is the finest convergence structure on $L(E, F)$ which agrees on $n T_{U}$ for every $n \in \mathbb{N}$ with the topology induced by $L_{s}(E, F)$. This means that the topology of $\left(L_{C}(E, F)\right)_{\tau}$ is the finest locally convex vector space topology agreeing on every member of $\left\{n T_{U}: n \in \mathbb{N}\right\}$ with the topology induced by $L_{s}(E, F)$. Since $\left\{n T_{U}: n \in \mathbb{N}\right\}$ is a countable set we can apply the following result of Roelcke (see [14]): the sets of the form

$$
W=T_{B_{0}} \cap \bigcap_{n \in N} \overline{\left(n T U^{+T} B_{n}\right)^{s}}
$$

where $B_{n}$ is a finite subset of $E$ for all $n \in \mathbb{N} \cup\{0\}$, are a O-neighborhood base for $\left(L_{C}(E, E)\right)_{\tau}$.

THEOREM III.3. If the set $K(E, E)$ of all compact operators from $E$ into $E$ is dense in $L_{c}(E, E)$ then we have for every Banach space $F$,

$$
\left(L_{c}(E, F)\right)_{\tau}=L_{c o}(E, F)
$$

Proof. Let

$$
W=T_{B_{0}} \cap \bigcap_{n \in \mathbb{N}} \overline{\left(n T U^{+T_{B}}\right)_{n}^{s}}
$$

be a 0-neighborhood in $\left(L_{c}(E, F)\right)_{\tau}$ where $B_{n}$ is a finite subset of $E$ for all $n \in \mathbb{N} \cup\{0\}$. Proposition III.2 implies the existence of a net $\left(T_{\alpha}\right)_{\alpha \in J}$ in $K(E, E)$ and of a real number $\eta>0$, such that $\left\|T_{\alpha}\right\| \leq z_{2} n$ for all $\alpha \in J$ and $\left(T_{\alpha}\right)_{\alpha \in J}$ converges to the identity mapping $I$ in $L_{c o}(E, E)$. Since $U \cap\left[B_{n}\right]$ is compact, where $\left[B_{n}\right]$ is the vector space generated by $B_{n}$ in $E$, we conclude from Lemma III.I: for every $n \in \mathbb{N}$ there exists a compact operator $S_{n} \in L(E, E)$ such that $\left\|S_{n}\right\| \leq n$ and $s_{n}(b)=b$ for all $b \in B_{n}$. From

$$
R=R \circ S_{n}+R\left(I-S_{n}\right) \text { for all } R \in n T_{S_{n}}(U)
$$


we conclude

$$
{ }^{T}(1 / n) S_{n}(U) \subseteq n T_{U}+T_{B}
$$

Defining

$$
K=\bigcup_{n \in \mathbb{N}}(1 / n) \overline{S_{n}(U)}
$$

we get

$$
T_{K \cup B}=T_{B_{0}} \cap n_{n \in N} T_{(1 / n) S_{n}(U)}=T_{B_{0}} \cap \prod_{n \in N} T_{(1 / n) S_{n}(U)} \subseteq W .
$$

Since $\overline{S_{n}(U)}$ is a compact subset of $n U$, the set $K \cup B_{0}$ is also compact, and Theorem III.3 is proved.

From Lemma II.l it follows immediately:

PROPOSITION III.4. A Banach space $E$ has the bounded approximation property if and only if the set $L E \otimes E$ of all operators of finite rank is dense in $\mathrm{L}_{\mathrm{C}}(E, E)$.

Together with Theorem III.3 this proposition implies:

THEOREM III.5. Assume $E$ is a Banach space with the bounded approximation property. Then we have $L_{c o}(E, F)=\left(L_{c}(E, F)\right)_{\tau}$ for every Banach space $F$.

\section{Nuclear spaces}

Let us remember that the bi-equicontinuous tensor product $L_{c o}(E) \otimes_{\varepsilon} F$ can be considered as a subspace of $L_{c o}(E, F)$. Now in some cases it is possible to show that the topology of $L_{c o}(E) \otimes_{\pi} F$ is finer than the topology induced by $\left(L_{c}(E, F)\right)_{\tau}$. If, in addition, $L E \otimes F$ is dense in $L_{c}(E, F)$ and $L_{c o}(E) \otimes_{\varepsilon} F=L_{c o}(E) \otimes_{\pi} F$, then we have $\left(L_{c}(E, F)\right)_{\tau}=L_{c o}(E, F)$. For this reason we examine nuclear spaces in this section. We will prove $\left(L_{c}(E, E)\right)_{\tau}=L_{c o}(E, F)$ if $E$ is a nuclear $(D F)$-space and $F$ is a Fréchet space or if $E$ is a nuclear Fréchet space and $F$ is a Banach space. Finally we will show 


$$
\left\{L_{c}\left(\mathbb{R}^{D}, z_{1}\right)\right]_{\tau} \neq L_{c o}\left(R^{D}, z_{1}\right)
$$

where $R^{D}$ is an uncountable product of $\mathbb{R}$ and $\tau_{1}$ is the Banach space of all absolutely convergent series.

PROPOSITION IV.1. Assume that on $L E \otimes F$ the topologies induced by $\left(L_{c}(E, F)\right)_{T}$ and $L_{c o}(E, F)$ agree and assume that $L E \otimes F$ is dense in $L_{\mathrm{c}}(E, F)$. Then we have

$$
\left(L_{c}(E, F)\right)_{\tau}=L_{c o}(E, F)
$$

Proof. For every closed 0-neighborhood $W$ in $\left(L_{c}(E, F)\right)_{\tau}$ there exist a precompact subset $K \subseteq E$ and a convex, circled 0 -neighborhood $V$ in $F$ such that

$$
\{g: g \in L E \otimes F, g(K) \subseteq V\} \subseteq W
$$

Let $T \in L(E, E)$ be an operator with $T(K) \subseteq \frac{2}{2} V$. Choose a filter $\Phi$ converging to $T$ in $L_{c}(E, F)$ which contains $L E \otimes F$. Since $\Phi$ converges to $T$ in $L_{c o}(E, F)$ one can find an $G \in \Phi$ with $(G-T)(K) \subseteq H^{\frac{1}{2}} V . \quad$ This implies $G \cap(L E \otimes F) \subseteq W$. Since $W$ is closed, $T$ belongs to $W$ and therefore we have proved $\left(L_{C}(E, F)\right)_{\tau}=L_{c o}(E, F)$.

LEMMA IV.2. The adherence $a(L E \otimes F)$, taken in $L_{c}(E, F)$, contains every nuclear mapping $T$ from $E$ into $F$.

Proof. For every nuclear mapping $T$ there exist an element $\lambda=\left(\lambda_{n}\right)_{n \in \mathbb{N}} \in Z_{1}$, an equicontinuous subset $\left\{\zeta_{n}: n \in \mathbb{N}\right\}$ of $L E$ and a bounded subset $\left\{y_{n}: n \in \mathbb{N}\right\}$ of $F$, such that

$$
T(x)=\sum_{n \in \mathbb{N}} \lambda_{n} \zeta_{n}(x) y_{n} \text { for all } x \in E
$$

Let $V$ be a convex, circled 0 -neighborhood in $F$ and let $x$ be an element of $E$. There exists an $r \in \mathbb{N}$ such that

$$
T(x)-\sum_{n=1}^{m} \lambda_{n} \zeta_{n}(x) y_{n} \subseteq \frac{2}{2} V \text { for all } m>r
$$


Mor eover there exist a 0 -neighborhood $U$ in $E$ and a real number $\mu>0$ such that $\zeta_{n}(U) \subseteq[-1,1]$ and $\mu y_{n} \in \frac{1}{2} V$ for all $n \in \mathbb{N}$. For each $m>r$ we have

$$
\begin{aligned}
T(x)-\sum_{n=1}^{m} \lambda_{n} \zeta_{n}\left(x+\mu\|\lambda\|^{-1} U\right) y_{n} & \subseteq \frac{3}{2} V-\sum_{n=1}^{m} \lambda_{n} \zeta_{n}\left(\mu\|\lambda\|^{-1} U\right) y_{n} \\
& \subseteq \frac{1}{2} V+\mu[-1,1] \sum_{n=1}^{m} \lambda_{n}\|\lambda\|^{-1} y_{n} \subseteq \frac{1}{2} V+\frac{3}{2} V \subseteq V .
\end{aligned}
$$

Therefore the sequence $\left(\sum_{n=1}^{m} \lambda_{n} \zeta_{n} \otimes y_{n}\right)_{m \in \mathbb{N}}$ converges to $T$ in $L_{c}(E, F)$.

To prove the next Iemma let us first mention a result of Brauner [4]: let $E$ be a $d F$-space and $F$ a Fréchet space. Then a subset $P$ is relatively compact in $L_{c o}(F, E)$ if and only if there exists a 0-neighborhood $U$ in $F$ such that $P(U)$ is relatively compact in $E$. Recall that $E$ is called a $d F$-space if and only if there exists a Fréchet space $H$ such that $E$ is isomorphic to $L_{C O}(H)$. Together with Lemma II.8 this result implies:

LEMMA IV.3. If $E$ is a $d F$-space and $F$ a Fréchet space then we have $\left(F \otimes_{\mathrm{C}} L_{\mathrm{c}} E\right)_{\tau}=F \otimes_{\pi} L_{\mathrm{co}}(E)$.

THEOREM IV.4. Let $E$ be a dF-space, $F$ a Fréchet space and assume that $E$ or $F$ is nuclear. Then

$$
\left(L_{c}(E, F)\right)_{\tau}=L_{c o}(E, F) .
$$

Proof. The mapping $\Theta: L_{c} E \times F \rightarrow L_{c}(E, F)$ defined by $[\theta(\zeta, y)](x)=\zeta(x) y$ for all $x \in E, y \in F$ and $\zeta \in L E$, is continuous. Therefore $\Theta$ induces a continuous mapping from $\left(L_{c} E \otimes_{c} F\right)_{\tau}=L_{c o}(E) \otimes_{\pi} F$ into $\left(L_{c}(E, F)\right)_{\tau}$. Since $E$ is nuclear if and only if $L_{C o}(E)$ is nuclear (see [4]) we get $L_{c o}(E) \otimes_{\pi} F=L_{c o}(E) \otimes_{\varepsilon} F$. But $L_{c o}(E) \otimes_{\varepsilon} F$ is a subspace of $L_{c o}(E, F)$ which implies that on $L E \otimes F$ the topologies induced by $\left(L_{C}(E, F)\right)_{\tau}$ and $L_{c o}(E, F)$ agree.

If $F$ is nuclear, Grothendieck has shown in [9], Chapter II, §2, 
no. 4, that every $T \in L(E, F)$ is nuciear. If $E$ is nuclear we argue as follows: by the preceding remark every $T \in L(E, F)$ is compact. Let $U$ be a convex, circled 0-neighborhood in $E$ such that $\overline{T(U)}$ is compact in $F$. Let $B$ be the Banach space $U_{n \in \mathbb{N}} n \overline{T(U)}$ with unit ball $\overline{T(U)}$. Now $T$ $n \in \mathbb{N}$

is a continuous mapping from $E$ into $B$. Since $E$ is nuclear, $T$ is a nuclear mapping from $E$ into $B$ and therefore also a nuclear mapping from $E$ into $F$. Proposition IV.I now implies $\left(L_{c}(E, E)\right)_{\tau}=L_{c o}(E, F)$.

We have shown in the proof of the corollary of Theorem II.7 that a nuclear $(D F)$-space is a $d F$-space. Therefore we get:

COROLLARY. Let $E$ be a nuclear (DF)-space and $F$ a Fréchet space. Then $\left(L_{c}(E, F)\right)_{\tau}=L_{c o}(E, F)$.

PROPOSITION IV.5. For every complete locally convex topological vector space $E$ and for every Banach space $F$ the topology of $L_{C O}(E) \otimes_{\pi} F$ is finer than the topology induced on $L E \otimes F$ by $\left(L_{c}(E, F)\right)_{\tau}$.

Proof. Let $U$ be the O-neighborhood filter in $E, B$ the family of all finite subsets of $E$ and $V=\{y: y \in F,\|y\| \leq 1\}$. For every subset $M \subseteq E$ define $T_{M}=\{T: T \in L(E, F), T(M) \subseteq V\}$. Let $W$ be a o-neighborhood in $\left(L_{C}(E, F)\right)_{\tau}$. For every $U \in U$ the filter generated by $\left\{T_{U \cup B}: B \in B\right\}$ converges to 0 in $L_{C}(E, F)$, which implies that, for every $U \in U$, there exists a $B_{U} \in B$ with ${ }^{T}{ }_{U B_{U}} \subseteq W$. Therefore the convex hull $C$ of $\underset{U \in U}{U} T_{U U B}$ is contained in $W$. Moreover, the convex hul1 $D$ of $U_{U \in U}\left(U \cup B_{U}\right)^{0}$ is a 0-neighborhood in $\left(L_{c}(E)\right)_{\tau}=L_{c o}(E)$ where the polars are taken with respect to the duality $\langle L E, E\rangle$. We will now show $D \otimes V \subseteq C \subseteq W$. Choose $\zeta \in D$ and $y \in V$. By definition of $D$ we have

$$
\zeta=\sum_{i=1}^{r} \lambda_{i} \zeta_{i}
$$


with $\zeta_{i} \in\left(U_{i} \cup B_{U_{i}}\right)^{0}, 0<\lambda_{i} \in \mathbb{R}$ for $1 \leq i \leq r$ and $\lambda_{1}+\ldots+\lambda_{r}=1$. Since

$$
\left(\zeta_{i} \otimes y\right)\left(U_{i} \cup B_{U_{i}}\right)=\zeta_{i}\left(U_{i} \cup B_{U_{i}}\right) y \subseteq[-1,1] y \subseteq V,
$$

we have $\left(\zeta_{i} \otimes y\right) \in T_{U_{i} \cup B_{U_{i}}}$ for $1 \leq i \leq r$ and

$$
\zeta \otimes y=\sum_{i=1}^{r} \lambda_{i}\left(\zeta_{i} \otimes y\right) \in C .
$$

THEOREM IV.6. If $E$ is a nuclear Fréchet space and $F$ a Banach space then $\left(L_{c}(E, F)\right)_{\tau}=L_{c o}(E, F)$.

Proof. By Proposition IV.5 the topology of $L_{C O}(E) \otimes_{\pi} E$ is finer than the topology induced by $\left(L_{c}(E, F)\right)_{\tau}$. Since $L_{c o}(E)$ is nuclear we have $L_{c o}(E) \otimes_{\pi} F=L_{c o}(E) \otimes_{\varepsilon} F$. Therefore the topologies induced on $L E \otimes F$ by $L_{c o}(E, F)$ and $\left(L_{c}(E, F)\right)_{\tau}$ agree. Since $E$ is nuclear, every $T \in L(E, F)$ is nuclear. Now Proposition IV.I and Lemma IV.2 imply $L_{c o}(E, F)=\left(L_{C}(E, F)\right)_{\tau}$.

In the following example we will show that there exist a complete, nuclear, barrelled space $E$ and a Banach space $F$ with $\left(L_{c}(E, F)\right)_{\tau} \neq L_{c O}(E, F)$. Therefore $L_{C O}(E, F)$ carries not always the strict topology generated by the topology of pointwise convergence on $L(E, F)$.

EXAMPLE. Let $D$ be an uncountable set and let $H$ be the family of all finite subsets of $D$. Define $R_{i}=\mathbf{R}$ for all $i \in D$. Let us denote by ind $L$ and ind $T$ the inductive limit taken in the category of all convergence spaces and in the category of all locally convex topological vector spaces respectively. Then we have

$$
L_{c}\left(\prod_{i \in D} R_{i}\right)=\operatorname{ind}_{H \in H^{\prime}} L_{c}\left(\prod_{i \in H} R_{i}\right)
$$

and therefore 


$$
L_{c o}\left(\prod_{i \in D} i\right)=\left\{L_{c}\left(\prod_{i \in D} R_{i}\right)\right\}_{\tau}=\operatorname{ind}_{H \in H} L_{c o}\left(\prod_{i \in H} R_{i}\right) .
$$

Since every $T \in L\left(\prod_{i \in D} R_{i}, z_{1}\right)$ has finite rank and since for every $H \in H$ we have

$$
L_{c}\left(\prod_{i \in H} R_{i}, z_{1}\right)=L_{c o}\left(\prod_{i \in H} R_{i}, z_{1}\right)=L_{c o}\left(\prod_{i \in H} R_{i}\right) \otimes_{\pi} i_{1}
$$

it is easy to see that

$$
L_{c}\left(\prod_{i \in D} R_{i}, z_{1}\right)=\operatorname{ind}_{H \in H^{L}} L_{c}\left(\prod_{i \in H} R_{i}, l_{1}\right)=\operatorname{ind}_{H \in H^{L}}\left(L_{c o}\left(\prod_{i \in H} R_{i}\right) \otimes_{\pi} z_{1}\right) \text {. }
$$

This implies

$$
\begin{aligned}
\left(L_{c}\left(\prod_{i \in D} R_{i}, z_{1}\right)\right)_{\tau}=i_{H \in H} T & \left(L_{c o}\left(\prod_{i \in H} R_{i}\right) \otimes_{\pi} z_{1}\right) \\
& =\left(\operatorname{ind}_{H \in H} L_{c o}\left(\prod_{i \in H} R_{i}\right)\right) \otimes_{\pi} z_{1}=L_{c o}\left(\prod_{i \in D} R_{i}\right) \otimes_{\pi} z_{1} .
\end{aligned}
$$

Since $L_{c o}\left(\mathbb{R}^{D}\right)$ is not nuclear, we have

$$
\left\{L_{c}\left(R^{D}, \tau_{1}\right)\right\}_{\tau}=L_{c o}\left(R^{D}\right) \otimes_{\pi} z_{I} \neq L_{c o}\left(R^{D}\right) \otimes_{\varepsilon} z_{I}=L_{c o}\left(R^{D}, z_{1}\right) .
$$

\section{References}

[1] Philippe Antoine, "Étude élémentaire des catégories d'ensembles structurEs", Bulz. Soc. Math. Belg. 18 (1966), 387-413.

[2] Ronald Beattie, "On the convergence vector space $L_{c}(E, F)$ and its dual space", Canad. Math. Bull. 21 (1978), 279-284.

[3] E. Binz und H.H. Keller, "Funktionenräume in der Kategorie der Limesräume", Ann. Acad. Sci. Fenn. Ser. A I Math. 383 (1966), $1-21$.

[4] Kalman Brauner, "Duals of Frechet spaces and a generalization of the Banach-Dieudonné theorem", Duke Math. J. 40 (1973), 845-855.

[5] H.-P. Butzmann, "Über die c-Reflexivität von $C_{c}(X)$ ", Comment. Math. Helv. 47 (1972), 92-101. 
[6] C.H. Cook and H.R. Fischer, "On equicontinuity and continuous convergence", Math. Am. 159 (1965), 94-104.

[7] H.R. Fischer, "Limesräume", Math. Ann. 137 (1959), 269-303.

[8] D.J.H. Garling, "A generalized form of inductive-limit topology for vector spaces", Proc. London Math. Soc. (3) 14 (1964), 1-28.

[9] Alexandre Grothendieck, Produits tensoriels topologiques et espaces nucléaires (Memoirs of the American Mathematical Society, 16. American Mathematical Society, Providence, Rhode Island, 1955).

[10] Hans Jarchow, "Marinescu-Räume", Comment. Math. Helv. 44 (1969), 138-163.

[11] W.B. Johnson, H.P. Rosenthal and M. Zippin, "On bases, finite dimensional decompositions and weaker structures in Banach spaces", Israel J. Math. 9 (1971), 488-506.

[12] Bernd Müller, "Vervollständigungen von Limesvektorräumen"

(Manuskripte, Fakultät für Mathematik und Informatik, Universität Mannheim, Mannheim, 1975).

[13] Bernd Müller, "T 3 -completions of convergence vector spaces", General topology and its relations to modem analysis and algebra IV, Part B, 298-307 (Proceedings of the Fourth Prague Topology Symposium, 1976. Society of Czechoslovak Mathematicians and Physicists, Prague, 1977).

[14] W. Roelcke, "On the finest locally convex topology agreeing with a given topology on a sequence of absolutely convex sets", Math. Ann. 198 (1972), 57-80.

[15] Helmut H. Schaefer, Topological vector spaces, Third printing corrected (Graduate Texts in Mathematics, 3. Springer-Verlag, New York, Heidelberg, Berlin, 1971).

[16] A. Wiweger, "Linear spaces with mixed topology", Studia Math. 20 (1961), 47-68.

Universität Mannheim,

Fakultät für Mathematik und Informatik, D-68 Mannheim,

Germany. 